AperTO - Archivio Istituzionale Open Access dell'Università di Torino

\title{
Prognostic Values of Multichannel Intraluminal Impedance and pH Monitoring in Newborns with Symptoms of Gastroesophageal Reflux Disease
}

\section{This is the author's manuscript}

Original Citation:

Availability:

This version is available http://hdl.handle.net/2318/124136

since

Published version:

DOI:10.1016/j.jpeds.2012.10.009

Terms of use:

Open Access

Anyone can freely access the full text of works made available as "Open Access". Works made available under a Creative Commons license can be used according to the terms and conditions of said license. Use of all other works requires consent of the right holder (author or publisher) if not exempted from copyright protection by the applicable law. 


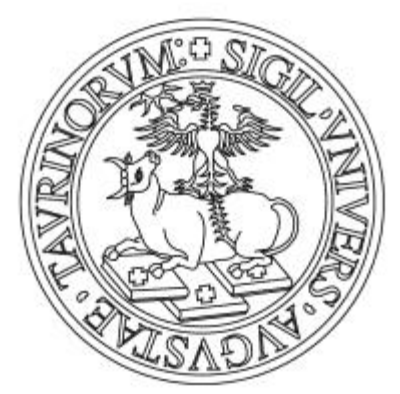

\section{UNIVERSITÀ DEGLI STUDI DI TORINO}

This Accepted Author Manuscript (AAM) is copyrighted and published by Elsevier. It is posted here by agreement between Elsevier and the University of Turin. Changes resulting from the publishing process - such as editing, corrections, structural formatting, and other quality control mechanisms - may not be reflected in this version of the text. The definitive version of the text was subsequently published in:

\section{[The journal of pediatrics, Vol. 36, Issue 4, April 2013, DOI: 10.1016/j.jpeds.2012.10.009]}

You may download, copy and otherwise use the AAM for non-commercial purposes provided that your license is limited by the following restrictions:

(1) You may use this AAM for non-commercial purposes only under the terms of the CC-BY-NCND license.

(2) The integrity of the work and identification of the author, copyright owner, and publisher must be preserved in any copy.

(3) You must attribute this AAM in the following format: Creative Commons BY-NC-ND license (http://creativecommons.org/licenses/by-nc-nd/4.0/deed.en), [+ Digital Object Identifier link to the published journal article on Elsevier's ScienceDirect ${ }^{\circledR}$ platform] 

with GERD Symptoms

Francesco Cresi ${ }^{1}$, Emanuela Locatelli ${ }^{1}$, Cristina Marinaccio ${ }^{2}$, Giulia Grasso ${ }^{3}$, Alessandra Coscia ${ }^{1}$ and Enrico Bertino ${ }^{1}$

${ }^{1}$ Neonatal Intensive Care Unit, S.Anna-Regina Margherita Children’s Hospital, Department of Pediatrics, University of Turin, Italy.

${ }^{2}$ Institute of Child Neuropsychiatry, S.Anna-Regina Margherita Children’s Hospital, University of Turin, Italy.

${ }^{3}$ Division of Pediatric Emergency, S. Anna -Regina Margherita Children’s Hospital, Turin, Italy.

\section{Address for correspondence:}

Dr. Francesco Cresi

Neonatal Intensive Care Unit

S.Anna-Regina Margherita Children’s Hospital

Department of Pediatrics, University of Turin

Piazza Polonia, 94 - 10126 Turin, Italy

Tel.: +390113135781

Fax: +390113134617

E-mail: francesco.cresi@unito.it

\section{Short title: Predicting Duration of GERD Symptoms in Newborns}

\section{Corresponding Author Information Reprint requests: Francesco Cresi}

Key Words: neonate; gastroesophageal reflux; esophageal dismotility; esopahgeal impedance; clinical practice; GER; GOR; GORD.

The authors have no conflicts of interest to disclose.

Francesco Cresi and Emanuela Locatelli wrote the first draft of the manuscript.

The authors have no financial relationships relevant to this article to disclose.

No honorarium, grant, or other form of payment was given to anyone to produce this manuscript.

Each author listed on the manuscript has seen and approved the submission of this version of the manuscript and takes full responsibility for the manuscript. 


\section{ABSTRACT}

Objective: To evaluate the relationship between multichannel intraluminal impedance and $\mathrm{pH}$ monitoring (MII/pH) values in newborns with symptoms of gastroesophageal reflux disease (GERD) and their clinical history in the first 3 years of life.

Patients and methods: Sixty-four newborns with GERD symptoms who underwent MII/pH in the first weeks of life were enrolled into a clinical follow-up program. Follow-up visits were programmed at 1, 3, 6, 9, 12, 18, 24 and 36 months. Patients were divided into three groups according to duration of symptoms: short (1-3 months), medium (4-9 months) and long (>9 months), and $\mathrm{MII} / \mathrm{pH}$ values were compared between these groups.

Results: Fifty-three patients completed the 3-year follow-up. The number of patients presenting with GERD symptoms decreased each month, following an exponential decay curve. When the $\mathrm{MII} / \mathrm{pH}$ values of the three lifetime symptom groups were compared, differences were found in the impedance bolus exposure index (IBEI) $(\mathrm{F}=83 ; \mathrm{p}=0.012)$ and proximal reflux frequency $(\mathrm{F}=410$; $\mathrm{p}=0.022$ ). These two MII variables showed an increasing trend from the short to the long lifetime symptom group. Weakly acidic reflux events, but not acid ones, were responsible for these differences.

Conclusion: $\mathrm{MII} / \mathrm{pH}$ in newborns has a prognostic value with regard to the duration of GERD symptoms and provides useful information that clinicians may give parents about the prognosis of symptomatic infants. IBEI and proximal reflux frequency seem to be the variables with the highest predictive value. Weakly acidic reflux events play an important role in determining the duration of GERD symptoms in newborns. 


\section{INTRODUCTION}

Gastroesophageal reflux (GER) can be considered physiologic in the first months of life. In newborns, a retrograde flow of gastric content in the esophagus can occur 2 or 3 times per hour and it is often accompanied by regurgitation or vomiting, but it can also be clinically silent.(1) GER tends to resolve spontaneously and it is often controlled by conservative management (position of infant after feedings, thickened or fractionated feedings). In approximately 5\% of newborns, GER can be associated with gastroesophageal reflux disease (GERD), characterized by different signs and symptoms influenced by the frequency and quality of reflux events.(2) Early diagnosis and treatment of GERD are important in neonatal intensive care units (NICUs), as the disease can worsen preterm conditions and lengthen hospitalization time.(3) Indeed, some authors have hypothesized a clinical correlation between GER and apneas of preterm newborns,(4) chronic respiratory diseases and pneumonia,(5) and feeding and growth problems.(6) This hypothesis has led to the pharmacological treatment of GERD in NICUs, both during hospitalization and at discharge, based on clinical diagnosis, even if that diagnosis is not supported by relevant exams. It has been recently reported that $25 \%$ of extremely low birth weight preterm newborns are discharged with drugs for GERD, with an extreme variability across different NICUs.(7) This is the result of the lack of guidelines and insufficient scientific information about GERD in newborns, and shows the need for more studies using new and more precise diagnostic techniques, such as multichannel intraluminal impedance and $\mathrm{pH}$ monitoring $(\mathrm{MII} / \mathrm{pH})$, which detects reflux events as changes in impedance recorded between pairs of electrodes located along a catheter in the esophageal lumen, and determines their $\mathrm{pH}$ by means of a $\mathrm{pH}$-sensitive antimony electrode.(8) $\mathrm{MII} / \mathrm{pH}$ allows us not only to evidence esophageal acid exposure, but also to detect non-acid or weakly acidic reflux events that are prevalent in newborns.(9) It was demonstrated that this technique is able to recognize a higher number of refluxes than traditional $\mathrm{pH}$-metry(10) and it is particularly suitable in newborns,(11) and its application in term and preterm newborns with GERD symptoms has been shown to be safe and well tolerated.(12) MII/pH allows for the observation of some distinctive 
features of reflux events in these patients, which could influence the corresponding clinical approach.(13) However, the use of MII/pH in clinical practice is currently limited in infants by the lack of data regarding the relationship between the examined MII/pH variables.

The aim of this study was to investigate the relationship between $\mathrm{MII} / \mathrm{pH}$ values recorded in a cohort of newborns with GERD symptoms, and their clinical outcome as evaluated by a 3-year clinical follow-up.

\section{MATERIALS AND METHODS}

Patients with clinical symptoms of GERD, as per criteria laid out in the North American Society for Pediatric Gastroenterology and Nutrition,(2) who were admitted to the neonatal care unit of the S.Anna-Regina Margherita Children’s Hospital from 2004 to 2008 and who underwent MII/pH, were consecutively enrolled into the study.

Included patients were term newborns in the first month of life or preterm newborns with a postmenstrual age of 36-46 weeks at MII/pH that had: 1) a valid MII/pH trace for a minimum of 19 consecutive hours, excluding artifacts; 2) absence of pharmacological therapy for GERD for at least 1 week before MII/pH; 3) a diet of breast milk or formula (thickened formulas were excluded); and 4) absence of infection, metabolic, or central nervous system diseases.

Written informed consent was obtained from the parents of all included patients. The research protocol was approved by the Ethical Committee of the S.Anna-Regina Margherita Children’s Hospital.

The Orenstein questionnaire was used as a guideline to prepare an anamnestic record for each patient,(14) which detailed the anthropometric data (weight, length, head circumference), symptoms, number and amount of meals, prior treatments and doses administered, and any diagnostic examinations performed.

After patients were discharged from the hospital, clinical follow-up visits were programmed at 1, 3, 6, 9, 12, 18, 24 and 36 months. Follow-up visits could be more frequent if the symptoms 
worsened, or if requested by the parents. At enrollment and during follow-up visits, patients were examined and measured, and the parents were administered the structured questionnaire which included questions on whether their infant was showing symptoms, if not when the symptoms stopped, and if so what the symptoms were and the corresponding therapy. A telephone interview was administered to parents if they were unable to bring their infant to a scheduled follow-up visit. We considered patients who were not present at two sequential follow-up visits, and those who presented with other chronic diseases during the follow-up period, to be lost to follow-up.

GERD symptoms were categorized as typical (excessive regurgitations, vomiting, blenching), atypical (crying, irritability, nocturnal waking and difficulties in feeding) and respiratory problems (apneas/desaturations/bradicardia, paleness, cyanosis, cough). Patients were assigned to one or more of these groups according to the symptoms they presented at enrollment.

In order to retrospectively evaluate the relationship between $\mathrm{MII} / \mathrm{pH}$ variables and duration of GERD symptoms, at the end of the follow-up period patients were classified into three lifetime symptom groups: those who presented a clinical condition suggestive of GERD for the first 3 months of life or less (short symptom group), the ones who presented this condition for 4-9 months (medium symptom group) and the ones who presented this condition for more than 9 months (long symptom group).

Z-scores of weight and height for age were calculated from the formula $\mathrm{Z}=\mathrm{x}-|\mathrm{X}| /|\mathrm{SD}|$, taking the CDC growth curves as reference at birth, at enrollment, at the end of symptoms and at the end of follow-up, to evaluate changes in anthropometric parameters independently from the age.

The characteristics of reflux events at enrollment were assessed by $\mathrm{MII} / \mathrm{pH}$ : a single-use $\mathrm{MII} / \mathrm{pH}$ intraesophageal probe with a pH-sensitive antimony electrode and seven integrated impedance electrodes was placed transnasally, with "containment" and "consolation" of the newborn, after a 3hour fasting period. The probe was tested and calibrated before insertion, and its distal $\mathrm{pH}$-sensitive tip was positioned $1.5 \mathrm{~cm}$ above the LES. The approximate position of the probe was calculated according to Strobel,(15) checked fluoroscopically and corrected if necessary. The catheter was 
connected to an exterior impedance device (Sleuth System, Sandhill Scientific Inc., Highlands Ranch, Colorado, USA) for signal processing and recording. Impedance was measured bipolarly between paired electrodes that formed six impedance channels. The distance between adjacent electrodes was $1.5 \mathrm{~cm}$. The total measuring segment extended from $1.5 \mathrm{~cm}$ above the LES (channel 1, distal) to the pharynx, with the $\mathrm{pH}$ sensor located at channel 1.

During $\mathrm{MII} / \mathrm{pH}$ recording patients were kept supine on a surface inclined at $30^{\circ}$ and received breast milk or formula from a feeding bottle every 4 hours. The MII/pH traces were visually evaluated by a single operator to avoid interobserver variability.(16) Segments with artifacts or interruptions of the signal were discarded. A MII reflux event was defined as a decrease of impedance starting in the most distal channel, extending proximally over two or more channels and followed by an increase in impedance to baseline values. The duration of a reflux event was defined as the time (in seconds) between its onset at the 50\% drop in impedance from baseline relative to nadir, and bolus exit at the 50\% recovery point from nadir to baseline recorded at the distal channel. Its proximal extent was defined as the number of channels sequentially involved in the temporary impedance decrease. A MII reflux event was defined as “proximal” when its proximal extent reached the most proximal MII-recording site, fluoroscopically located in the pharynx. The reflux $\mathrm{pH}$ was the nadir esophageal $\mathrm{pH}$ recorded during the event. According to these values, the reflux events were divided into acidic ( $\mathrm{pH}<4)$, weakly acidic ( $\mathrm{pH} 4-7)$ and weakly alkaline ( $\mathrm{pH}>7)$. A $\mathrm{pH}$-metry reflux event was defined as a temporary fall of the $\mathrm{pH}$ signal under 4 longer than 5 seconds.

$\mathrm{MII} / \mathrm{pH}$ traces were analyzed by evaluating seven different parameters:

MII-reflux frequency (reflux events/hour);

MII-reflux duration (seconds);

MII-reflux proximal extent (number of channels);

MII-proximal reflux frequency (reflux events/hour); 
MII-IBEI (\%), calculated as the total percentage of time with bolus reflux detected by impedance;

pH-metry reflux frequency (reflux events/hour);

pH-metry reflux index (RI \%), calculated as the total percentage of time with bolus reflux detected by pH-metry.

Upon discharge from the hospital it was recommended that all parents put their newborns to sleep on an inclined plane, on their back, close to the parents' bed, and that parents not smoke and try to deliver meals slowly. Subjects who had a positive pH-metry (RI \% >5) were prescribed pharmacological therapy with H2-blockers. In these patients, parents were asked to stop treatment 2 weeks before each clinical follow-up visit in order to perform symptom assessment, and to resume treatment only if their child's symptoms worsened.

\section{Statistical analysis}

The distribution of all the continuous variables was assessed by the Shapiro-Wilk test and by graphic analysis. Date regarding growth in the three groups were evaluated by considering differences in $\mathrm{Z}$ score ( $\Delta \mathrm{Z}$ score) between anthropometric values at birth, at enrolment, at the end of symptoms and at the end of follow-up. $\Delta$ Z-score and MII/pH variables between different groups of patients were evaluated with ANOVA and post hoc Tukey test was used to evaluate the pair-wise significance. The Odd Ratio (OR) between MII/pH values and lifetime symptoms was evaluated using as cut-off the limits of the 95\% confidence interval of each MII/pH variable.

$\mathrm{p}<0.05$ was considered statistically significant. All results are shown as mean and standard deviation (SD) unless otherwise indicated. Statistical analysis was performed using the STAT software package for Windows, release 5.5 (StatSoft, Inc. Tulsa, Oklahoma, USA).

\section{RESULTS}


Sixty-four newborns were enrolled into the study, and 53 of them completed the 3-year followup. Eleven patients were lost to follow-up: eight because they were not present at two sequential follow-up visits and the three others because they were diagnosed with congenital pathologies after enrollment (Di George syndrome, Hirshprung disease and cystic fibrosis).

The 53 newborns (18 preterm, 35 term) who completed the follow-up had a mean \pm SD gestational age of $37.3 \pm 3.0$ weeks, and a birth weight and length of $2758.3 \pm 681$ grams and $46.5 \pm 4.7$ $\mathrm{cm}$, respectively. At $\mathrm{MII} / \mathrm{pH}$ they had a mean age of $28.9 \pm 17.3$ days, with a postmenstrual age of $41.4 \pm 2.5$ weeks, a weight of $3395.1 \pm 696.3$ grams and a length of $51.9 \pm 2.7 \mathrm{~cm}$.

At enrollment, 44 newborns presented typical GERD symptoms, 34 presented atypical symptoms and 45 had respiratory problems. Seven (13.2\%) newborns presented only one category of symptoms, 24 (45.3\%) had symptoms belonging to two different categories and 22 (41.5\%) had symptoms from all three defined categories of GERD symptoms.

The mean GERD symptoms duration was 6.4 \pm 6.8 months (median 4.5; inter-quartile range 0.335.4). The number of patients presenting with GERD symptoms decreased during the follow-up period, following an exponential decay curve: at 3 months 44 (83\%) patients displayed GERD symptoms, but only 18 patients (34.0\%) displayed symptoms at 6 months, 13 (24.5\%) at 9 months, $10(18.9 \%)$ at 12 months, $3(5.7 \%)$ at 18 months, two (3.8\%) at 24 months and one at 36 months.

Typical GERD symptoms were predominant in the first 3-4 months, and completely disappeared at 24 months; atypical symptoms became predominant between 4 and 24 months, and completely disappeared at 30 months. Respiratory problems showed a rapid decline between 1 and 6 months, due to a strong reduction in apneas, desaturation and bradicardia, but they became predominant in the infants still symptomatic after 26 months for the appearance of chronic cough after 18 months (Figure 1).

During a total of 1273.1 hours of MII/pH, 3175 reflux events (608 acid, 2567 weakly acidic) were detected by MII. Their mean \pm SD frequency per patient was $2.6 \pm 0.9$ per hour. The mean duration was $24.9 \pm 8.7$ seconds. The mean proximal extent was $4.5 \pm 0.5$ channels. The mean $\mathrm{pH}$ was 
5.0 \pm 0.6 and the mean IBEI\% was $1.8 \pm 0.9$. The number of proximal reflux events was 2283 (71.90\%) with a mean frequency per patient of $1.9 \pm 0.8$ reflux events per hour.

A total of 1271 reflux events were detected by $\mathrm{pH}$-metry. The mean $\pm \mathrm{SD}$ reflux frequency per patient was $1.1 \pm 0.7$, and the RI\% was $3.1 \pm 2.3$. Only 10 infants had a positive $\mathrm{pH}$-metry $(\mathrm{RI} \%>5$ ) without significant differences for the other $\mathrm{MII} / \mathrm{pH}$ variables compared to the other patients, and were discharged with a prescription for of H2-blockers. Two of them were treated for 1 month, 1 for 2-3 months, 2 for 4-6 months and 5 for more than 6 months. All of them achieved partial improvement of symptoms during pharmacological treatment.

We compared MII/pH values between patients who presented clinical symptoms at enrollment from one, two, or three of the categories defined in this study, and were not found statistical differences between values for all the MII and $\mathrm{pH}$ variables.

Nineteen patients were assigned to the short lifetime symptom group, 22 to the medium group and 12 to the long group.

There were no differences between the three groups for age, gestational age, postmenstrual age and anthropometric measures at $\mathrm{MII} / \mathrm{pH}$ (Table 1).

$\Delta$ Z-score for weight between short, medium and long lifetime symptom groups were respectively $0.24 \pm 0.55 ;-0.25 \pm 0.47$ and $-0.19 \pm 0.56(\mathrm{p}=0.95, \mathrm{~F}=0.05)$ between birth and enrollment; $0.04 \pm 0.29$; $0.22 \pm 0.67$ and $-0.45 \pm 0.58(\mathrm{p}=0.02, \mathrm{~F}=4.30 ; \mathrm{p}=0.04$ short vs. long group; $\mathrm{p}=0.02$ medium vs. long group) between enrollment and the end of symptoms; $0.58 \pm 0.83 ; 0.67 \pm 1.12$ and $0.54 \pm 0.58(\mathrm{p}=0.91$, $\mathrm{F}=0.09$ ) between the end of symptoms and the end of follow-up.

$\Delta \mathrm{Z}$-score for height between short, medium and long lifetime symptom groups were respectively $0.46 \pm 0.92 ; 0.62 \pm 0.87$ and $0.52 \pm 1.08(\mathrm{p}=0.86, \mathrm{~F}=0.15)$ between birth and enrollment; $0.73 \pm 0.38$; $0.41 \pm 0.30$ and $0.18 \pm 0.25(\mathrm{p}=0.88, \mathrm{~F}=0.13$ ) between enrollment and the end of symptoms; $0.02 \pm 0.48 ; 0.11 \pm 0.36$ and $0.13 \pm 0.35(\mathrm{p}=0.72, \mathrm{~F}=0.33)$ between the end of symptoms and the end of follow-up. 
When comparing the MII/pH values of the three groups of patients, differences were found in IBEI $(\mathrm{p}=0.012, \mathrm{~F}=4.835)$ and proximal reflux frequency $(\mathrm{p}=0.022, \mathrm{~F}=4.105)$. These two MII variables showed a progressive increasing trend from the short to the long symptom group. No differences were found between the three groups for the other MII variables, or for $\mathrm{pH}$ variables (Table 1).

The 95\% CI limits for IBEI and proximal reflux frequency were 1.58-2.10 and 1.68-2.13 respectively. On a total of 24 patients with IBEI lower than the lower 95\% CI limit, 22 (91.7\%) resolved symptoms in the first 9 months (OR 5.8; CI 1.13-29.77; $\mathrm{p}=0.036$ ), whereas 8/18 (44.4\%) patients with IBEI greater than the upper 95\% CI limit presented symptoms over 9 months (OR 6.2; CI 1.54-25.04; $\mathrm{p}=0.001)$.

On a total of 24 patients with proximal reflux frequency lower than the lower 95\% CI limit 21(87.5\%) resolved symptoms in the first 9 months (OR 3.15; CI 0.74-13.33; $\mathrm{p}=0.19$ ), whereas 8/19 (42.1\%) patients with proximal reflux frequency greater than the upper 95\% CI limit presented symptoms over 9 months (OR 0.18; CI 0.46-0.732; p=0.016).

Finally, we evaluated the two significant MII variables, IBEI and proximal reflux frequency, separately for acid and weakly acidic reflux events in the three lifetime symptom groups (Figure 2). We observed that weakly acidic reflux events, but not acid reflux events, were responsible for the significant differences observed between the three groups of patients for both MII variables.

\section{DISCUSSION}

$\mathrm{MII} / \mathrm{pH}$ is a novel technique to detect GER in newborns and infants.(13) The reliability of $\mathrm{MII} / \mathrm{pH}$ in detecting reflux events and its ability to characterize them according to duration, proximal extent and $\mathrm{pH}$ make this technique excellent for clinical research; it has already been used in newborns to assess the relationship between apnea of preterm and GER,(4),(17),(18) the effects of drugs(19),(20) and thickening agents.(21) However, the lack of reference values and the incomplete knowledge of the clinical significance of the majority of measured variables limit its use in clinical practice. The present study represents, to our knowledge, the first attempt to investigate 
the relationship between $\mathrm{MII} / \mathrm{pH}$ values assessed in the first weeks of life in infants with GERD symptoms, and clinical outcome, with 3 years of follow-up.

During follow-up, GERD symptoms tended to disappear spontaneously, following a decay curve in which half of the patients were asymptomatic in the fifth month of age, and the vast majority were asymptomatic after the first year of life according with what was demonstrated in a previous prospective study.(22)

Analysis of $\mathrm{MII} / \mathrm{pH}$ variables demonstrated that, as already observed by other authors, in the first weeks of life the frequency of reflux events is physiologically high (2-4 reflux events/hour) and that weakly acidic reflux events are predominant.(9) We also observed a high proximal reflux frequency, which represented more than two-thirds of the total reflux events.

The most relevant result was the demonstration of a relationship between two of the MII/pH variables and lifetime symptoms. In particular, we observed that the short symptom group (who were asymptomatic 3 months after enrollment) had a significantly lower IBEI and proximal reflux frequency compared with the long symptoms group (whose symptoms were resolved in 4-6 months).

In our sample, the patients with IBEI values and proximal reflux frequency respectively lower than 1.51 and 1.68 were related with a high probability to have a duration of symptoms shorter than 9 months, whereas the patients with IBEI values greater than 2.10 were related with a high probability to have a duration of symptoms longer than 9 months.

Furthermore, the patients of the long symptom group presented a slower weight growth between the disappearance of symptoms and the enrollment. These results suggest that high values of IBEI and proximal reflux frequency revealed by $\mathrm{MII} / \mathrm{pH}$ could be predictive of prolonged duration of symptoms and failure in weight growth.

It is well known that $\mathrm{MII} / \mathrm{pH}$ values are influenced by age. A limit of the present study was to consider a sample of patients formed by both preterm and term newborns. Differences in gestational age among our patients might have weakened our results by increasing the variability of $\mathrm{MII} / \mathrm{pH}$ 
and anthropometric values, but did not invalidate the differences between the three lifetime symptoms groups that were homogeneous regarding age, gestational age, weight and length at enrolment.

The second important result emerged from the analysis of the relationship between the MII variables and duration of GERD symptoms by acid and weakly acidic reflux events separately. Indeed, weakly acidic reflux events, but not acid reflux events, were significant in determining the differences in IBEI and proximal reflux frequency in the three different lifetime symptom groups.

It should be noted that neither of the two variables obtained from $\mathrm{pH}$-metry alone (pH-reflux frequency and RI), were useful in identifying lifetime symptom groups. This finding shows that $\mathrm{MII} / \mathrm{pH}$ is more accurate than $\mathrm{pH}$-metry alone to study GERD in the first weeks of life,(23) in agreement with the hypothesis that the $\mathrm{pH}$-metry alone is not sufficient to study GER in newborns because of the prevalence of weakly acidic reflux events due to the frequent consumption of milk, which buffers gastric acidity. These findings support the hypothesis that weakly acidic reflux events have a key role in determining GERD symptoms in newborns and suggest that the role of H2blockers and proton pump inhibitors in the first months of life should be reconsidered. In fact, these drugs have been described as inefficient in newborns(24),(25) and potentially harmful to preterm newborns.(26)

\section{CONCLUSION}

$\mathrm{MII} / \mathrm{pH}$ performed in first weeks of life has a prognostic value for duration of GERD symptoms. IBEI and proximal reflux frequency seem to be the variables with the highest predictive value. Weakly acidic reflux events play an important role in determining the duration of GERD symptoms in newborns. These results represent useful information that clinicians may give parents with regard to prognosis, and they offer the first reference values of MII/pH in symptomatic newborns, which could be useful in interpreting these values in clinical practice. 
Abbreviations: GER - gastroesophageal reflux; GERD - gastroesophageal reflux disease; IBEI impedance bolus exposure index; $\mathrm{MII} / \mathrm{pH}$ - multichannel intraluminal impedance and $\mathrm{pH}$ monitoring; NICU - neonatal intensive care unit; RI - reflux index; SD - standard deviation; LES lower esophageal sphincter. 


\section{REFERENCES}

1. Poets CF. Gastroesophageal reflux: a critical review of its role in preterm infants. Pediatrics 2004;113:e128-132.

2. Rudolph CD, Mazur LJ, Liptak GS, Baker RD, Boyle JT, Colletti RB, Gerson WT, Werlin SL. Guidelines for evaluation and treatment of gastroesophageal reflux in infants and children: recommendations of the North American Society for Pediatric Gastroenterology and Nutrition. J. Pediatr. Gastroenterol. Nutr 2001;32 Suppl 2:S1-31.

3. Khalaf MN, Porat R, Brodsky NL, Bhandari V. Clinical correlations in infants in the neonatal intensive care unit with varying severity of gastroesophageal reflux. J. Pediatr. Gastroenterol. Nutr 2001;32:45-9.

4. Corvaglia L, Zama D, Gualdi S, Ferlini M, Aceti A, Faldella G. Gastro-oesophageal reflux increases the number of apnoeas in very preterm infants. Arch. Dis. Child. Fetal Neonatal Ed 2009;94:F188-192.

5. Weir K, McMahon S, Barry L, Ware R, Masters IB, Chang AB. Oropharyngeal aspiration and pneumonia in children. Pediatr. Pulmonol. 2007;42:1024-31.

6. Bhatia J, Parish A. GERD or not GERD: the fussy infant. J Perinatol 2009;29 Suppl 2:S7-11.

7. Malcolm WF, Gantz M, Martin RJ, Goldstein RF, Goldberg RN, Cotten CM. Use of medications for gastroesophageal reflux at discharge among extremely low birth weight infants. Pediatrics 2008;121:22-7.

8. Trachterna M, Wenzl TG, Silny J, Rau G, Heimann G. Procedure for the semi-automatic detection of gastro-oesophageal reflux patterns in intraluminal impedance measurements in infants. Med Eng Phys 1999;21:195-201.

9. López-Alonso M, Moya MJ, Cabo JA, Ribas J, del Carmen Macías M, Silny J, Sifrim D. Twenty-four-hour esophageal impedance-pH monitoring in healthy preterm neonates: rate and characteristics of acid, weakly acidic, and weakly alkaline gastroesophageal reflux. Pediatrics 2006;118:e299-308. 
10. Francavilla R, Magistà AM, Bucci N, Villirillo A, Boscarelli G, Mappa L, Leone G, Fico S, Castellaneta S, Indrio F, Lionetti E, Moramarco F, Cavallo L. Comparison of esophageal pH and multichannel intraluminal impedance testing in pediatric patients with suspected gastroesophageal reflux. J. Pediatr. Gastroenterol. Nutr. 2010;50:154-60.

11. Wenzl TG, Skopnik H. Intraluminal impedance: an ideal technique for evaluation of pediatric gastroesophageal reflux disease. Curr Gastroenterol Rep 2000;2:259-64.

12. Peter CS, Wiechers C, Bohnhorst B, Silny J, Poets CF. Detection of small bolus volumes using multiple intraluminal impedance in preterm infants. J. Pediatr. Gastroenterol. Nutr 2003;36:381-4.

13. Vandenplas Y, Rudolph CD, Di Lorenzo C, Hassall E, Liptak G, Mazur L, Sondheimer J, Staiano A, Thomson M, Veereman-Wauters G, Wenzl TG, North American Society for Pediatric Gastroenterology Hepatology and Nutrition, European Society for Pediatric Gastroenterology Hepatology and Nutrition. Pediatric gastroesophageal reflux clinical practice guidelines: joint recommendations of the North American Society for Pediatric Gastroenterology, Hepatology, and Nutrition (NASPGHAN) and the European Society for Pediatric Gastroenterology, Hepatology, and Nutrition (ESPGHAN). J. Pediatr. Gastroenterol. Nutr 2009;49:498-547.

14. Kleinman L, Rothman M, Strauss R, Orenstein SR, Nelson S, Vandenplas Y, Cucchiara S, Revicki DA. The infant gastroesophageal reflux questionnaire revised: development and validation as an evaluative instrument. Clin. Gastroenterol. Hepatol. 2006;4:588-96.

15. Strobel CT, Byrne WJ, Ament ME, Euler AR. Correlation of esophageal lengths in children with height: application to the Tuttle test without prior esophageal manometry. J. Pediatr. 1979;94:81-4.

16. Loots CM, van Wijk MP, Blondeau K, Dalby K, Peeters L, Rosen R, Salvatore S, Wenzl TG, Vandenplas Y, Benninga MA, Omari TI. Interobserver and intraobserver variability in pH- 
impedance analysis between 10 experts and automated analysis. J. Pediatr. 2012;160:441446.e1.

17. Slocum C, Arko M, Di Fiore J, Martin RJ, Hibbs AM. Apnea, bradycardia and desaturation in preterm infants before and after feeding. J Perinatol 2009;29:209-12.

18. Di Fiore JM, Arko M, Whitehouse M, Kimball A, Martin RJ. Apnea is not prolonged by acid gastroesophageal reflux in preterm infants. Pediatrics 2005;116:1059-63.

19. Cresi F, Marinaccio C, Russo MC, Miniero R, Silvestro L. Short-term effect of domperidone on gastroesophageal reflux in newborns assessed by combined intraluminal impedance and $\mathrm{pH}$ monitoring. J Perinatol 2008;28:766-70.

20. Corvaglia L, Spizzichino M, Zama D, Aceti A, Mariani E, Legnani E, Faldella G. Sodium Alginate (Gaviscon ${ }^{\circledR}$ ) does not reduce apnoeas related to gastro-oesophageal reflux in preterm infants. Early Hum. Dev. 2011;87:775-8.

21. Corvaglia L, Ferlini M, Rotatori R, Paoletti V, Alessandroni R, Cocchi G, Faldella G. Starch thickening of human milk is ineffective in reducing the gastroesophageal reflux in preterm infants: a crossover study using intraluminal impedance. J. Pediatr 2006;148:265-8.

22. Hegar B, Dewanti NR, Kadim M, Alatas S, Firmansyah A, Vandenplas Y. Natural evolution of regurgitation in healthy infants. Acta Paediatr 2009;98:1189-93.

23. Loots CM, Benninga MA, Davidson GP, Omari TI. Addition of pH-impedance monitoring to standard pH monitoring increases the yield of symptom association analysis in infants and children with gastroesophageal reflux. J. Pediatr. 2009;154:248-52.

24. Wheatley E, Kennedy KA. Cross-over trial of treatment for bradycardia attributed to gastroesophageal reflux in preterm infants. J. Pediatr. 2009;155:516-21.

25. Salvatore S, Hauser B, Salvatoni A, Vandenplas Y. Oral ranitidine and duration of gastric pH $>4.0$ in infants with persisting reflux symptoms. Acta Paediatr. 2006;95:176-81. 
26. Terrin G, Passariello A, De Curtis M, Manguso F, Salvia G, Lega L, Messina F, Paludetto R, Canani RB. Ranitidine is associated with infections, necrotizing enterocolitis, and fatal outcome in newborns. Pediatrics 2012;129:e40-45. 
Figure 1. Frequency of GERD symptoms by symptom category during follow-up: (A) absolute frequencies; (B) normalized frequencies.

Notes: typical symptoms include excessive regurgitations, vomiting, blenching; atypical symptoms include crying, irritability, nocturnal waking and difficulties in feeding; respiratory problems include apneas/desaturations/bradicardia, paleness, cyanosis, cough.

Figure 2. IBEI (A) and proximal reflux frequency (B) values of acid and weakly acidic reflux events in short, medium and long lifetime symptom groups. Data are presented as mean (dots), SD (boxes) and range (whiskers). 